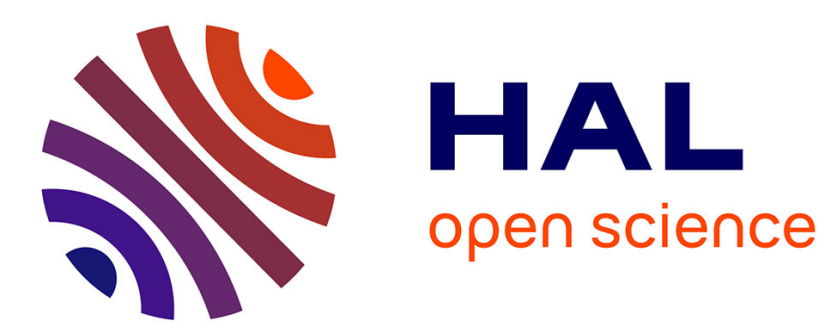

\title{
Twenty Parameters Families of Solutions to the NLS Equation and the Eleventh Peregrine Breather
}

\author{
Pierre Gaillard, Mickael Gastineau
}

\section{To cite this version:}

Pierre Gaillard, Mickael Gastineau. Twenty Parameters Families of Solutions to the NLS Equation and the Eleventh Peregrine Breather. Communications in Theoretical Physics, 2016, 65 (2), pp.136-144. 10.1088/0253-6102/65/2/136 . hal-01224526v2

\section{HAL Id: hal-01224526 \\ https://hal.science/hal-01224526v2}

Submitted on 12 Dec 2015

HAL is a multi-disciplinary open access archive for the deposit and dissemination of scientific research documents, whether they are published or not. The documents may come from teaching and research institutions in France or abroad, or from public or private research centers.
L'archive ouverte pluridisciplinaire HAL, est destinée au dépôt et à la diffusion de documents scientifiques de niveau recherche, publiés ou non, émanant des établissements d'enseignement et de recherche français ou étrangers, des laboratoires publics ou privés. 


\title{
The eleventh Peregrine breather and twenty parameters families of solutions to the NLS equation.
}

\author{
${ }^{+}$Pierre Gaillard, ${ }^{\times}$Mickaël Gastineau \\ + Institut de Mathématiques de Bourgogne, \\ 9 Av. Alain Savary, Dijon, France : Dijon, France : \\ e-mail: Pierre.Gaillard@u-bourgogne.fr, \\ × ASD, IMCCE-CNRS UMR8028, Observatoire de Paris, UPMC, \\ 77 Av. Denfert-Rochereau, 75014 Paris, France : \\ e-mail: gastineau@imcce.fr
}

December 12, 2015

\begin{abstract}
The Peregrine breather of order eleven $\left(P_{11}\right.$ breather $)$ solution to the focusing one dimensional nonlinear Schrödinger equation (NLS) is explicitly constructed here. Deformations of the Peregrine breather of order 11 with 20 real parameters solutions to the NLS equation are also given : when all parameters are equal to 0 we recover the famous $P_{11}$ breather. We obtain new families of quasi-rational solutions to the NLS equation in terms of explicit quotients of polynomials of degree 132 in $x$ and $t$ by a product of an exponential depending on $t$. We study these solutions by giving patterns of their modulus in the $(x ; t)$ plane, in function of the different parameters.
\end{abstract}

PACS : 35Q55, 37K10, 4710A, 47.35.Fg, 47.54.Bd.

Keywords : NLS equation, wronskians, Peregrine breather, rogue waves. 


\section{Introduction}

The story of the nonlinear Schrödinger equation (NLS) begins with the works of Zakharov and Shabat in 1968. It was solved in 1972 by using the inverse scattering method $[1,2]$. The first quasi-rational solution to NLS equation was constructed in 1983 by Peregrine [3], nowadays called worldwide Peregrine breather. Akhmediev, Eleonski and Kulagin obtained the two-phase almost periodic solution to the NLS equation and obtained the first higher order analogue of the Peregrine breather $[4,5]$ in 1986; other families of higher order 3 and 4 were constructed in a series of articles by Akhmediev et al. $[6,7]$, using Darboux transformations. Since the beginning of the years 2010, there was a renewed interest for this equation and many works were published using various methods. In 2011, the solutions to the NLS equation were presented as a quotient of two wronskians in $[8,9]$. In 2012, Guo, Ling and Liu constructed another representation of the solutions to the NLS equation, as a quotient of two determinants [10] using the generalized Darboux transformation.

Ohta and Yang presented in [11] solutions to the NLS equation by means of determinants, using a new approach based on the Hirota bilinear method. Then in 2013, it was found in [12], solutions expressed in terms of determinants of order $2 N$ depending on $2 N-$ 2 real parameters; the complete proof was recently given in [13]. A new representation has been found as a ratio of a determinant of order $N+1$ by another one of order $N$ by Ling and Zhao in [14]. Very recently in 2014, another approach have been given in [15] using a dressing method where the solutions are expressed as the quotient of a determinant of order $N+1$ by another one of order $N$.

With the method given in the present work, we construct new solutions to the focusing one dimensional nonlinear Schrödinger equation which appear as deformations of the (analogue) Peregrine breather of order 11 with 20 real parameters : when all the parameters are equal to 0 , we recover the famous $P_{11}$ breather. These solutions are completely expressed as a quotient of two polynomials of degree 132 in $x$ and $t$ by an exponential depending on $t$. We do not have the space to present them here; we only present plots in the $(x ; t)$ plane to analyze the evolution of the solutions in function of the different parameters.

\section{Determinant represen- tation of solutions to NLS equation}

We use in the following to construct deformations of the $P_{11}$ breather, solutions to the NLS equation, the results obtained in $[9,12]$.

Theorem 2.1 The function $v$ defined by

$v(x, t)=\frac{\operatorname{det}\left(\left(n_{j k}\right)_{j, k \in[1,2 N]}\right)}{\operatorname{det}\left(\left(d_{j k}\right)_{j, k \in[1,2 N]}\right)} e^{(2 i t-i \varphi)}$

is a quasi-rational solution to the NLS equation

$$
i v_{t}+v_{x x}+2|v|^{2} v=0,
$$


where

$$
\begin{aligned}
& n_{j 1}=f_{j, 1}(x, t, 0), \\
& n_{j k}=\frac{\partial^{2 k-2} f_{j, 1}}{\partial \epsilon^{2 k-2}}(x, t, 0), \\
& n_{j N+1}=f_{j, N+1}(x, t, 0), \\
& n_{j N+k}=\frac{\partial^{2 k-2} f_{j, N+1}}{\partial \epsilon^{2 k-2}}(x, t, 0), \\
& d_{j 1}=g_{j, 1}(x, t, 0), \\
& d_{j k}=\frac{\partial^{2 k-2} g_{j, 1}}{\partial \epsilon^{2 k-2}}(x, t, 0), \\
& d_{j N+1}=g_{j, N+1}(x, t, 0), \\
& d_{j N+k}=\frac{\partial^{2 k-2} g_{j, N+1}}{\partial \epsilon^{2 k-2}}(x, t, 0), \\
& 2 \leq k \leq N, 1 \leq j \leq 2 N
\end{aligned}
$$

The functions $f$ and $g$ are defined for $1 \leq k \leq N$ by :

$$
\begin{aligned}
& f_{4 j+1, k}=\gamma_{k}^{4 j-1} \sin A_{k}, \\
& f_{4 j+2, k}=\gamma_{k}^{4 j} \cos A_{k}, \\
& f_{4 j+3, k}=-\gamma_{k}^{4 j+1} \sin A_{k}, \\
& f_{4 j+4, k}=-\gamma_{k}^{4 j+2} \cos A_{k}, \\
& f_{4 j+1, N+k}=\gamma_{k}^{2 N-4 j-2} \cos A_{N+k}, \\
& f_{4 j+2, N+k}=-\gamma_{k}^{2 N-4 j-3} \sin A_{N+k}, \\
& f_{4 j+3, N+k}=-\gamma_{k}^{2 N-4 j-4} \cos A_{N+k}, \\
& f_{4 j+4, k}=\gamma_{k}^{2 N-4 j-5} \sin A_{N+k}, \\
& g_{4 j+1, k}=\gamma_{k}^{4 j-1} \sin B_{k}, \\
& g_{4 j+2, k}=\gamma_{k}^{4 j} \cos B_{k}, \\
& g_{4 j+3, k}=-\gamma_{k}^{4 j+1} \sin B_{k}, \\
& g_{4 j+4, k}=-\gamma_{k}^{4 j+2} \cos B_{k}, \\
& g_{4 j+1, N+k}=\gamma_{k}^{2 N-4 j-2} \cos B_{N+k}, \\
& g_{4 j+2, N+k}=-\gamma_{k}^{2 N-4 j-3} \sin B_{N+k}, \\
& g_{4 j+3, N+k}=-\gamma_{k}^{2 N-4 j-4} \cos B_{N+k}, \\
& g_{4 j+4, N+k}=\gamma_{k}^{2 N-4 j-5} \sin B_{N+k},
\end{aligned}
$$

The arguments $A_{\nu}$ and $B_{\nu}$ of these functions are given for $1 \leq \nu \leq 2 N$ by

$$
\begin{aligned}
& A_{\nu}=\kappa_{\nu} x / 2+i \delta_{\nu} t-i x_{3, \nu} / 2-i e_{\nu} / 2, \\
& B_{\nu}=\kappa_{\nu} x / 2+i \delta_{\nu} t-i x_{1, \nu} / 2-i e_{\nu} / 2 .
\end{aligned}
$$

The terms $\kappa_{\nu}, \delta_{\nu}, \gamma_{\nu}$ are defined by $1 \leq \nu \leq 2 N$

$$
\begin{aligned}
& \kappa_{j}=2 \sqrt{1-\lambda_{j}^{2}}, \delta_{j}=\kappa_{j} \lambda_{j}, \\
& \gamma_{j}=\sqrt{\frac{1-\lambda_{j}}{1+\lambda_{j}}}, \kappa_{N+j}=\kappa_{j}, \\
& \delta_{N+j}=-\delta_{j}, \gamma_{N+j}=1 / \gamma_{j}, \\
& 1 \leq j \leq N,
\end{aligned}
$$

where $\lambda_{j}$ are given for $1 \leq j \leq N$ by :

$$
\lambda_{j}=1-2 j^{2} \epsilon^{2}, \lambda_{N+j}=-\lambda_{j} .
$$

The terms $x_{r, \nu}(r=3,1)$ are defined for $1 \leq \nu \leq 2 N$ by :

$$
x_{r, \nu}=(r-1) \ln \frac{\gamma_{\nu}-i}{\gamma_{\nu}+i} .
$$

The parameters $e_{\nu}$ are given by

$$
\begin{aligned}
& e_{j}=i \sum_{k=1}^{N-1} \tilde{a}_{j} \epsilon^{2 k+1} j^{2 k+1} \\
& -\sum_{k=1}^{N-1} \tilde{b}_{j} \epsilon^{2 k+1} j^{2 k+1} \\
& e_{N+j}=i \sum_{k=1}^{N-1} \tilde{a}_{j} \epsilon^{2 k+1} j^{2 k+1} \\
& +\sum_{k=1}^{N-1} \tilde{b}_{j} \epsilon^{2 k+1} j^{2 k+1} \\
& 1 \leq j \leq N
\end{aligned}
$$

\section{Quasi-rational solutions of order 11 with twenty parameters}

We construct here deformations of the

(1 Peregrine breather $P_{11}$ of order 11 depending on 20 parameters. This construction is based on Theorem 2.1. This theorem in various formulations was completely shown in the articles published previously $[8,9,12,13]$.

To obtain the quasi rational solutions of equation NLS one uses the functions $f$ and $g$ defined previously by (1). One carries out limited developments of these functions in $\epsilon$ and their derivatives with respect to $\epsilon$, to order $2 N$ if one wants the solutions with the order $N$. The calculation of the two determinants given in theorem 2.1 then gives the polynomials searched in the expression of the solutions.

We do not give the analytic expression of the solution to NLS equation of order 11 with twenty parameters because of the length of the expression. The computations were done using the 
computer algebra systems Maple and TRIP [16]. For simplicity, we denote

$$
\begin{aligned}
& d_{3}:=\operatorname{det}\left(\left(n_{j k)_{j, k \in[1,2 N]}}\right),\right. \\
& d_{1}:=\operatorname{det}\left(\left(d_{j k)_{j, k \in[1,2 N]}}\right) .\right.
\end{aligned}
$$

The number of terms of the polynomials of the numerator $d 3$ and denominator $d 1$ of the solutions are shown in the table below (Table 1) when other $a_{i}$ and $b_{i}$ are set to 0 . The computation of $d_{3}\left(a_{1}, b_{1}, x, t\right)$ and $d_{1}\left(a_{1}, b_{1}, x, t\right)$ requires 13 days on a 32 -cores computer.

\begin{tabular}{|c|c|}
\hline $\mathbf{N}=\mathbf{1 1}$ & $\begin{array}{c}\text { Number } \\
\text { of terms }\end{array}$ \\
\hline$d_{3}\left(a_{1}, b_{1}, x, t\right)$ & 803534 \\
\hline$d_{1}\left(a_{1}, b_{1}, x, t\right)$ & 407850 \\
\hline$d_{3}\left(a_{2}, b_{2}, x, t\right)$ & 306417 \\
\hline$d_{1}\left(a_{2}, b_{2}, x, t\right)$ & 155543 \\
\hline$d_{3}\left(a_{3}, b_{3}, x, t\right)$ & 165321 \\
\hline$d_{1}\left(a_{3}, b_{3}, x, t\right)$ & 83925 \\
\hline$d_{3}\left(a_{4}, b_{4}, x, t\right)$ & 105667 \\
\hline$d_{1}\left(a_{4}, b_{4}, x, t\right)$ & 53637 \\
\hline$d_{3}\left(a_{5}, b_{5}, x, t\right)$ & 74720 \\
\hline$d_{1}\left(a_{5}, b_{5}, x, t\right)$ & 37930 \\
\hline$d_{3}\left(a_{6}, b_{6}, x, t\right)$ & 56409 \\
\hline$d_{1}\left(a_{6}, b_{6}, x, t\right)$ & 28638 \\
\hline$d_{3}\left(a_{7}, b_{7}, x, t\right)$ & 44491 \\
\hline$d_{1}\left(a_{7}, b_{7}, x, t\right)$ & 22590 \\
\hline$d_{3}\left(a_{8}, b_{8}, x, t\right)$ & 35459 \\
\hline$d_{1}\left(a_{8}, b_{8}, x, t\right)$ & 17999 \\
\hline$d_{3}\left(a_{9}, b_{9}, x, t\right)$ & 26282 \\
\hline$d_{1}\left(a_{9}, b_{9}, x, t\right)$ & 13332 \\
\hline$d_{3}\left(a_{10}, b_{10}, x, t\right)$ & 15049 \\
\hline$d_{1}\left(a_{10}, b_{10}, x, t\right)$ & 7632 \\
\hline
\end{tabular}

Table 1: Number of terms for the polynomials $d_{3}$ and $d_{1}$ of the solutions to the NLS equation.

We construct figures to show deformations of the eleventh Peregrine breather. We get different types of symmetries in the plots in the $(x, t)$ plane.
We give some examples of this fact in the following discussion. The present study follows works on order $N=3$ to $N=10$ given in $[17,18,19,20,21,22$, $23,24]$.

\subsection{Peregrine breather of or- der 11}

If we choose $\tilde{a}_{i}=\tilde{b}_{i}=0$ for $1 \leq i \leq 10$, we obtain the classical eleventh Peregrine breather

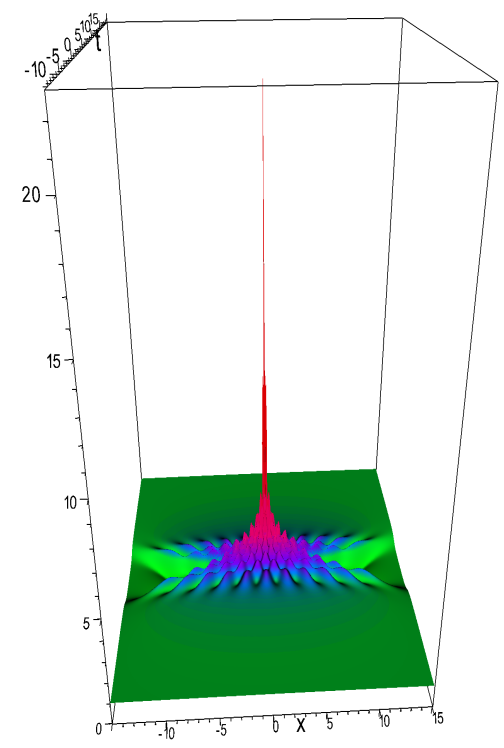

Figure 1: Solution of NLS, N=11, all parameters equal to 0 , Peregrine breather $P_{11}$.

\subsection{Variation of parameters}

With other choices of parameters, we obtain all types of configurations : triangles and multiple concentric rings configurations with a maximum of 66 peaks. 

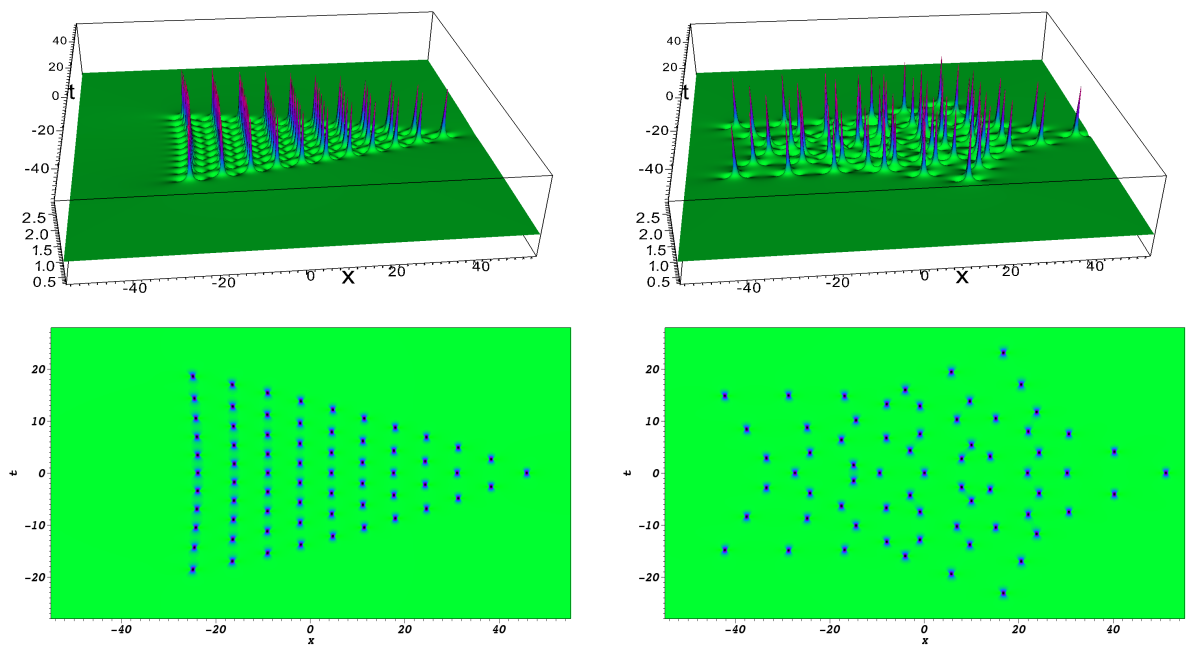

Figure 2: Solution of NLS, N=11, $\tilde{a}_{1}=$ $10^{3}$ : triangle with 66 peaks; in bottom, sight of top.

Figure 4: Solution of NLS, N=11, $\tilde{a}_{2}=$ $10^{5}$ : 9 rings with $5 ; 10 ; 10 ; 5 ; 5 ; 10 ; 5$ : $10 ; 5$ peaks, with in the center one peak; in bottom, sight of top.
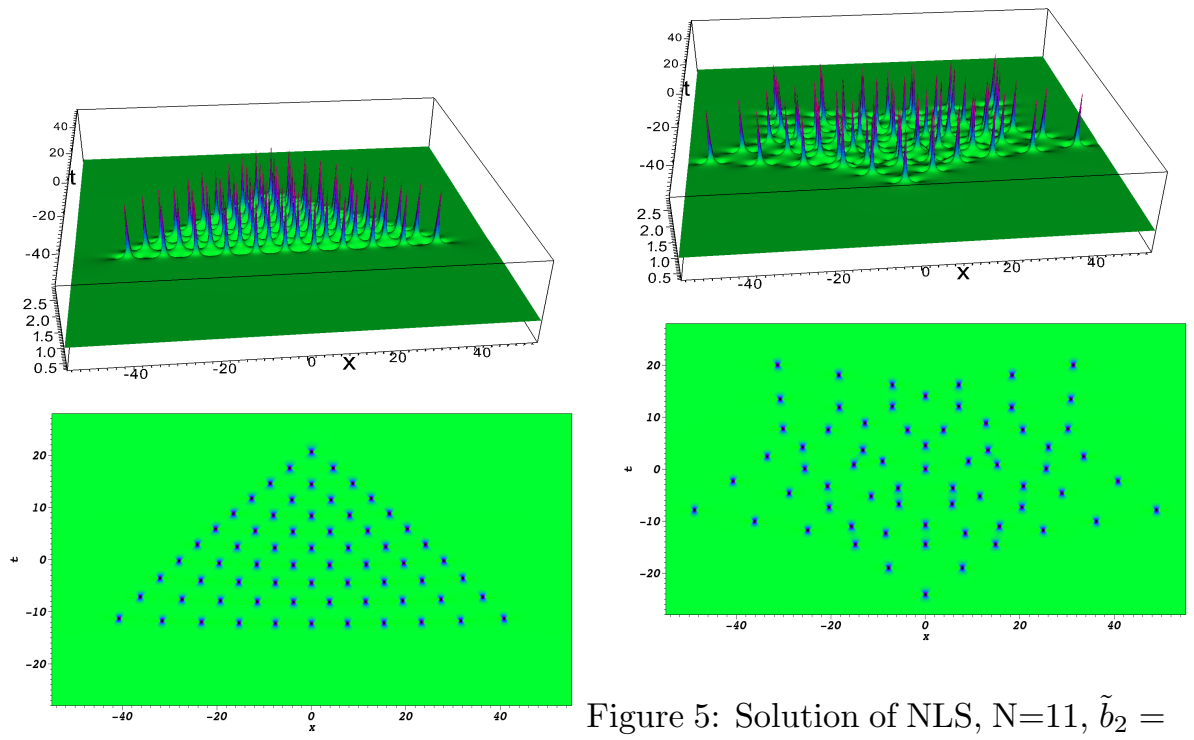

Figure 5: Solution of NLS, N=11, $\tilde{b}_{2}=$ $10^{5}$ : 9 rings with $5 ; 10 ; 10 ; 5 ; 5 ; 10 ; 5$ :

Figure 3: Solution of NLS, N=11, $\tilde{b}_{1}=$ $10^{3}$ : triangle with 66 peaks; in bot$10 ; 5$ peaks, with in the center one tom, sight of top. 

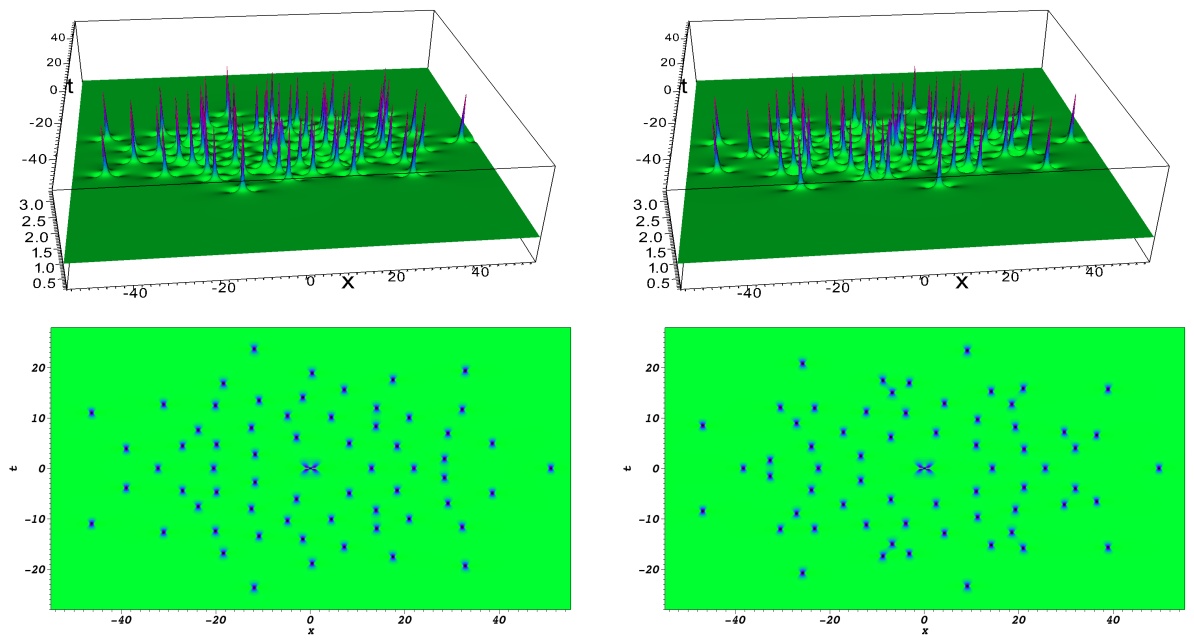

Figure 6: Solution of NLS, N=11, $\tilde{a}_{3}=$ $10^{7}$ : 7 rings with $7 ; 14 ; 7 ; 14 ; 7 ; 7 ; 7$ peaks, with in the center $P_{2}$; in bottom, sight of top.

Figure 8: Solution of NLS, N=11, $\tilde{a}_{4}=$ $10^{9}$ : 6 rings with $9 ; 9 ; 18 ; 9 ; 9 ; 9$ peaks, with in the center $P_{2}$; in bottom, sight of top.
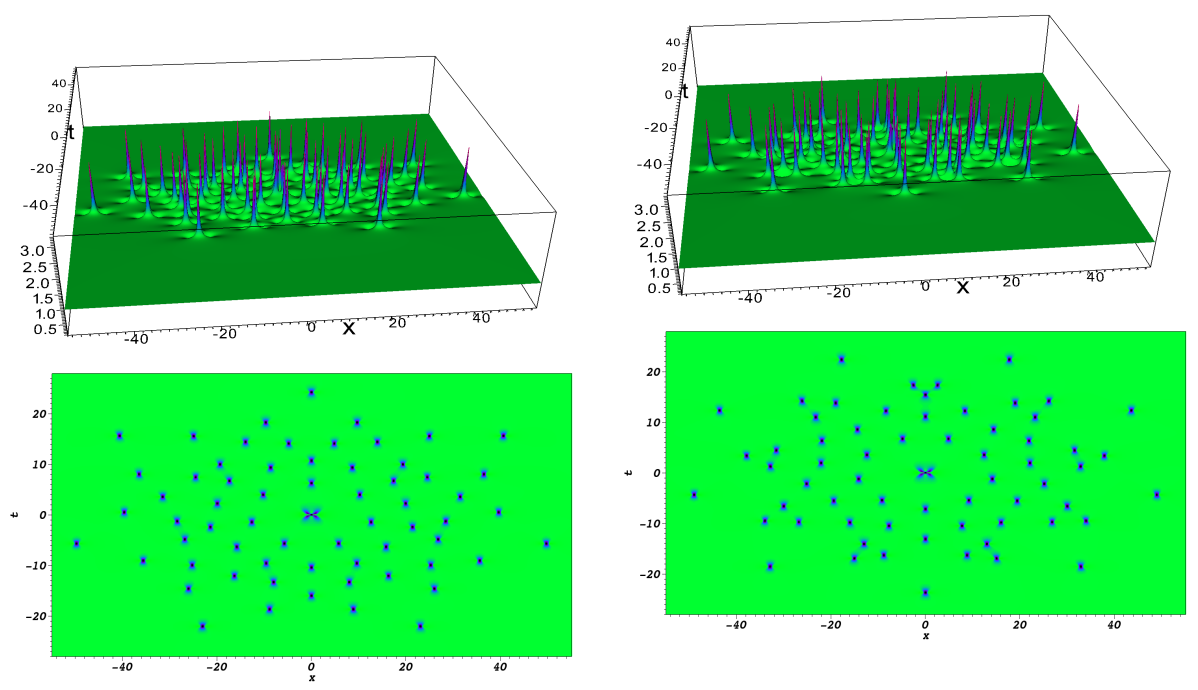

Figure 7: Solution of NLS, N=11, $\tilde{b}_{3}=$ $10^{7}: 7$ rings with $7 ; 14 ; 7 ; 14 ; 7 ; 7 ; 7$ peaks, with in the center $P_{2}$; in bottom, sight of top.

Figure 9: Solution of NLS, N=11, $\tilde{b}_{4}=$ $10^{9}: 6$ rings with $9 ; 9 ; 18 ; 9 ; 9 ; 9$ peaks, with in the center $P_{2}$; in bottom, sight of top. 

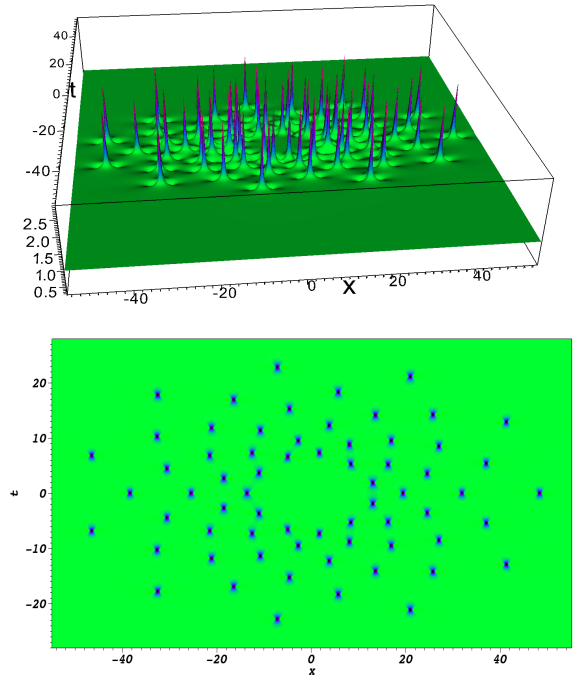

Figure 10: Solution of NLS, $N=11$, $\tilde{a}_{5}=10^{11}: 6$ rings of 11 peaks without a central peak; in bottom, sight of top.
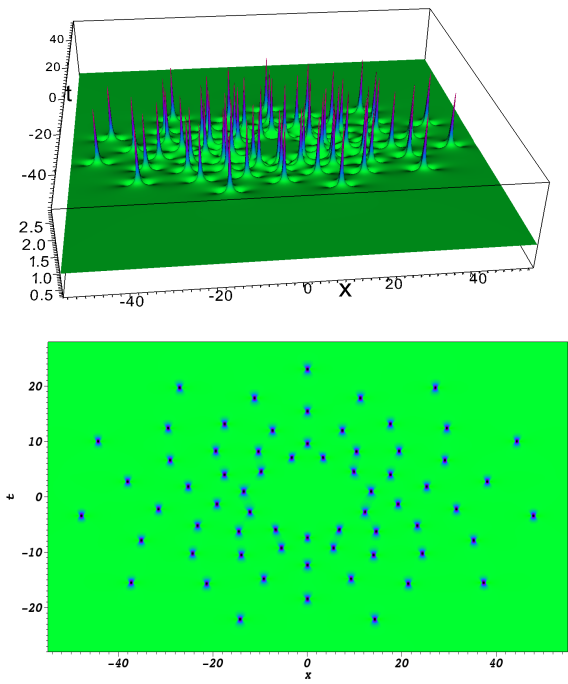

Figure 11: Solution of NLS, $N=11$, $\tilde{b}_{5}=10^{11}: 6$ rings of 11 peaks without a central peak; in bottom, sight of top.
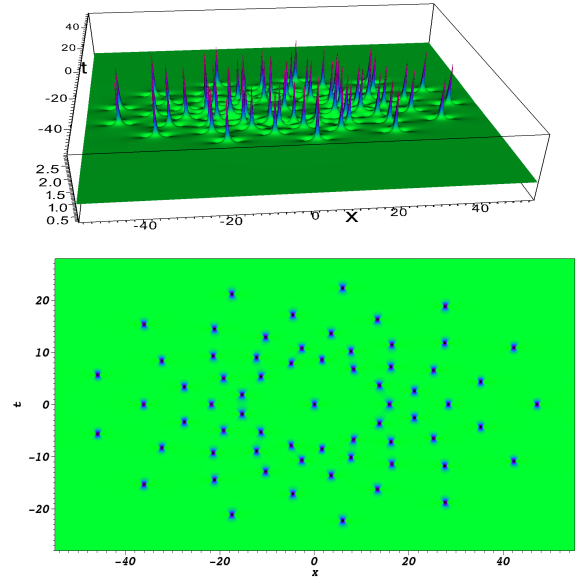

Figure 12: Solution of NLS, N=11, $\tilde{a}_{6}=10^{13}: 5$ rings with 13 peaks and in the center one peak; in bottom, sight of top.
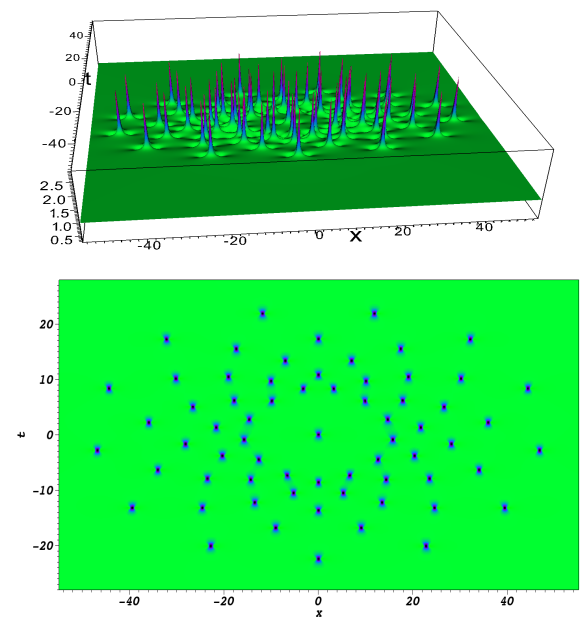

Figure 13: Solution of NLS, $N=11$, $\tilde{b}_{6}=10^{13}: 5$ rings with 13 peaks and in the center one peak; in bottom, sight of top. 

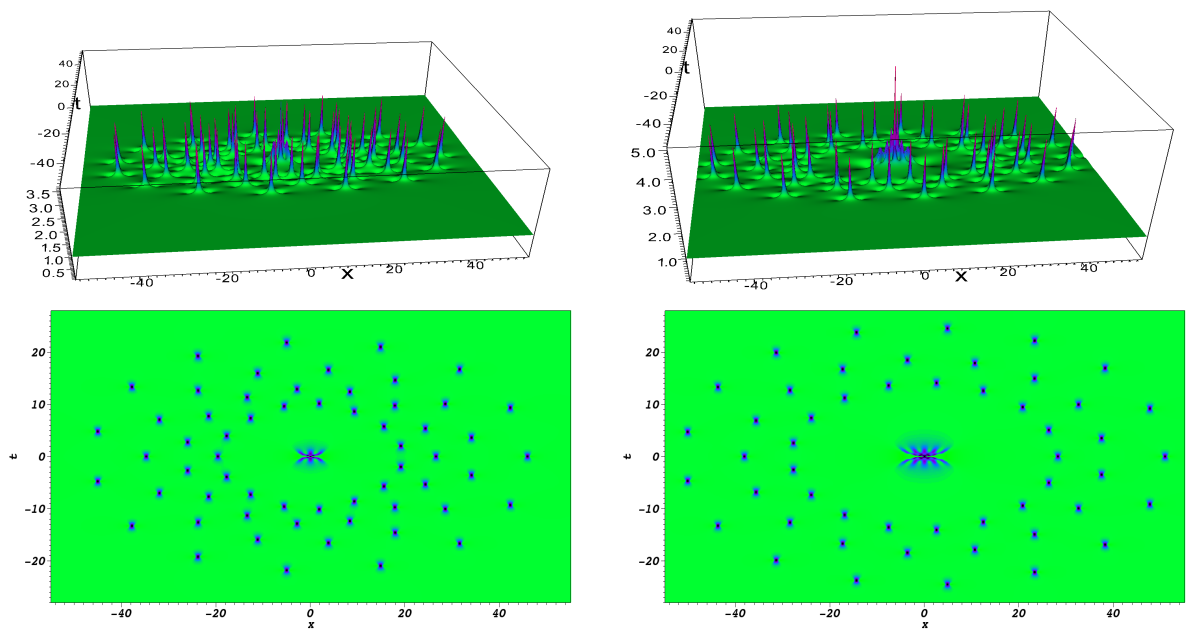

Figure 14: Solution of NLS, N=11, $\tilde{a}_{7}=10^{15}: 4$ rings with 15 peaks and in the center the Peregrine breather of order 3 ; in bottom, sight of top.
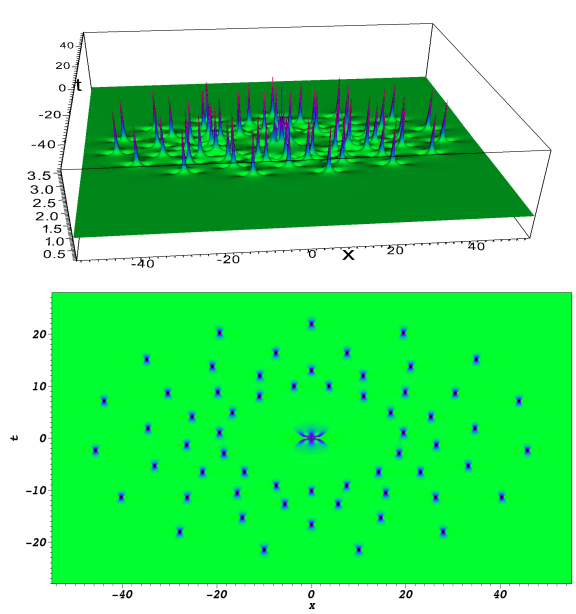

Figure 15: Solution of NLS, N=11, $\tilde{b}_{7}=10^{15}: 4$ rings with 15 peaks and in the center the Peregrine breather of order 3 ; in bottom, sight of top.
Figure 16: Solution of NLS, N=11, $\tilde{a}_{8}=10^{18}: 3$ rings with 17 peaks and in the center the Peregrine breather of order 5 ; in bottom, sight of top.
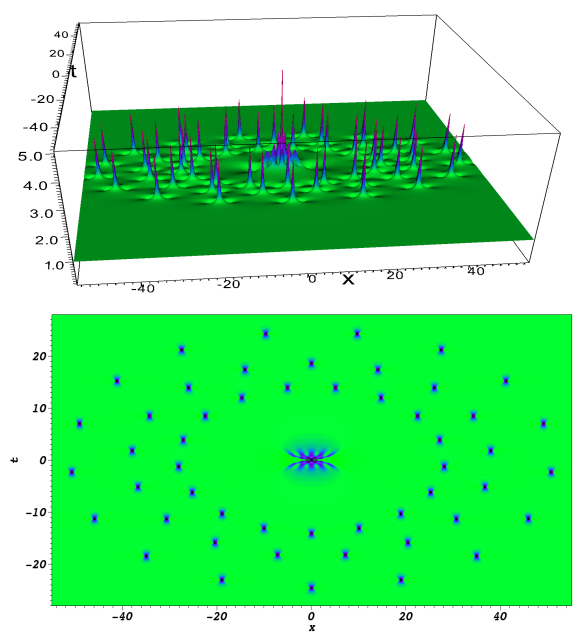

Figure 17: Solution of NLS, $N=10$, $\tilde{b}_{8}=10^{18}: 3$ rings with 17 peaks and in the center the Peregrine breather of order 5 ; in bottom, sight of top. 

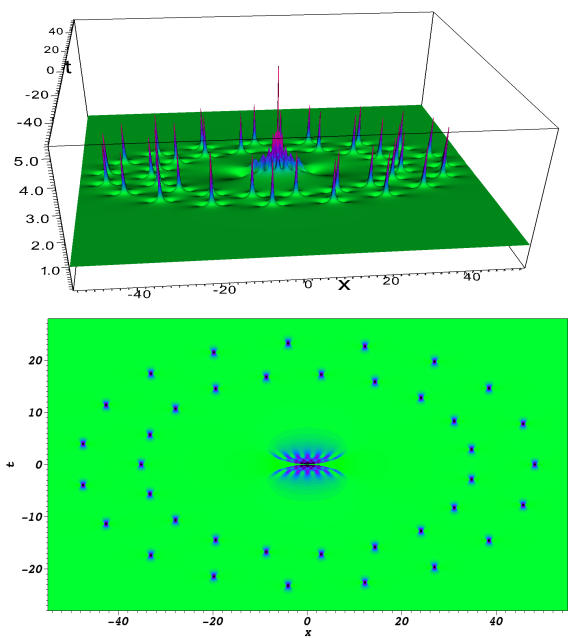

Figure 18: Solution of NLS, $\mathrm{N}=10$, $\tilde{a}_{9}=10^{20}:$ two rings with 19 peaks and in the center the Peregrine breather of order 7; in bottom, sight of top.
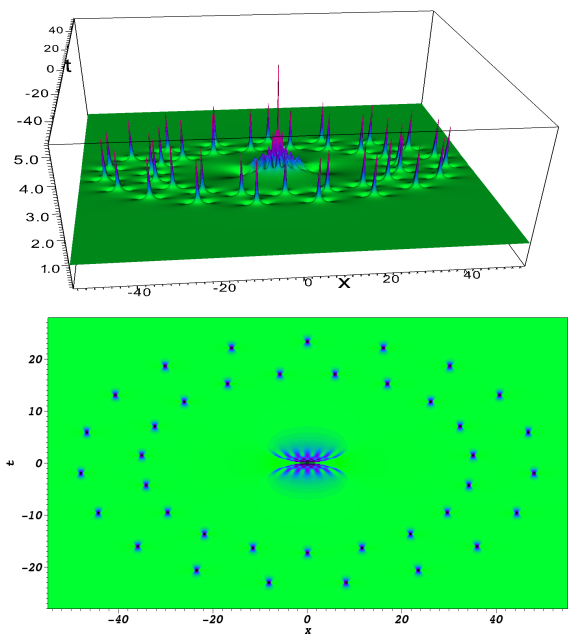

Figure 19: Solution of NLS, $N=10$, $\tilde{b}_{9}=10^{20}$ : two rings with 19 peaks and in the center the Peregrine breather of order 7; in bottom, sight of top.
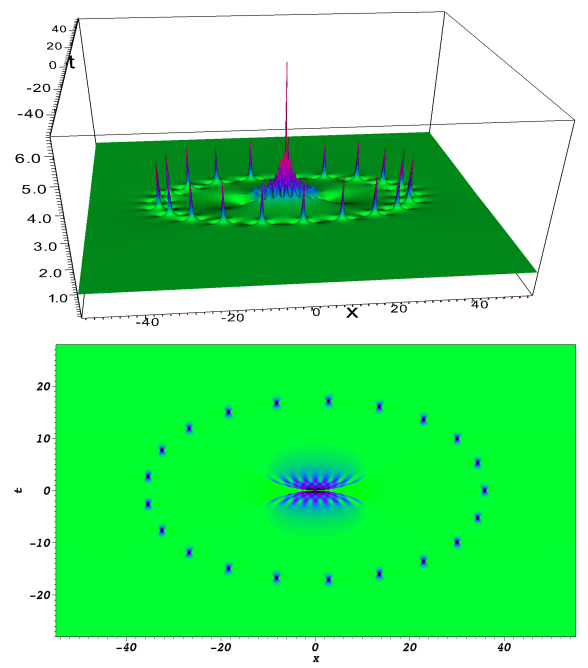

Figure 20: Solution of NLS, $N=10$, $\tilde{a}_{10}=10^{20}:$ one ring with 21 peaks and in the center the Peregrine breather of order 9; in bottom, sight of top.
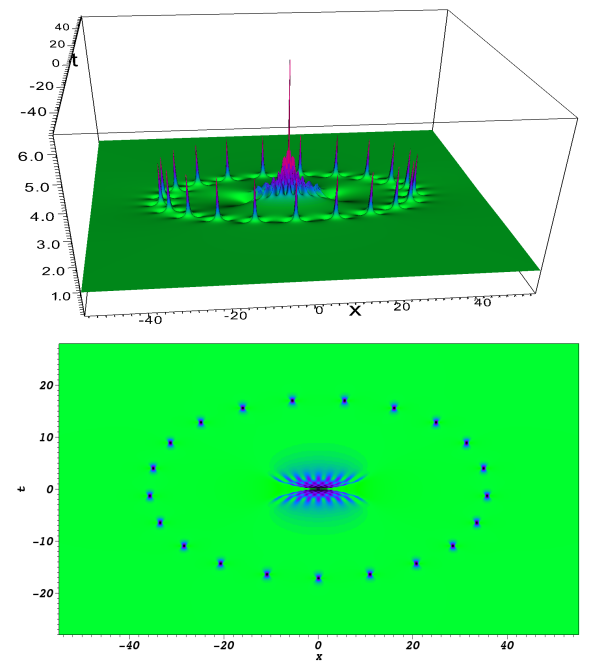

Figure 21: Solution of NLS, $N=10$, $\tilde{b}_{10}=10^{20}$ : one ring with 21 peaks and in the center the Peregrine breather of order 9; in bottom, sight of top. 


\section{Conclusion}

We have constructed explicitly solutions to the NLS equation of order 11 with 20 real parameters. The explicit representation in terms of polynomials of degree 132 in $x$ and $t$ is obtained. His expression is too large to be published in this text. It is the first time that the Peregrine breather of order eleven with its deformations with twenty parameters is presented to our knowledge. It confirms the property about the shape of the breather in the $(x, t)$ coordinates, the maximum of amplitude equal to $2 N+1$ and the degree of polynomials in $x$ and $t$ here equal to $N(N+1)$.

We obtained different patterns in the $(x ; t)$ plane by different choices of these parameters. So we obtain a classification of the rogue waves at order 11.

It is fundamental to note the similar role played by $a_{i}$ and $b_{i}$ for the same given index $i$ : one obtains exactly the same structures of the modulus of the solutions to NLS equation in the $(x ; t)$ plan.

In the cases $a_{1} \neq 0$ or $b_{1} \neq 0$ we obtain triangles with a maximum of 66 peaks; for $a_{2} \neq 0$ or $b_{2} \neq 0$, we have 9 rings with respectively $5,10,10,5,5,10,5$, 10,5 peaks with in the center one peak. For $a_{3} \neq 0$ or $b_{3} \neq 0$, we obtain 7 rings with respectively $7,14,7,14,7,7,7$ peaks with in the center the Peregrine $P_{2}$. For $a_{4} \neq 0$ or $b_{4} \neq 0$, we have 7 rings with 9 peaks on each of them with in the center the Peregrine $P_{2}$. For $a_{5} \neq 0$ or $b_{5} \neq 0$, we have 6 rings of 11 peaks on each of them without a central peak. For $a_{6} \neq 0$ or $b_{6} \neq 0$, we have 5 rings with 13 peaks on each of them and in the center one peak. For $a_{7} \neq 0$ or $b_{7} \neq 0$, we have 4 rings with 15 peaks on each of them and in the center the Peregrine breather of order
3. For $a_{8} \neq 0$ or $b_{8} \neq 0$, we have 3 rings with 17 peaks on each of them and in the center the Peregrine breather of order 5 . For $a_{9} \neq 0$ or $b_{9} \neq 0$, we have 2 rings with 19 peaks and in the center the Peregrine breather of order 7 . At least, for $a_{10} \neq 0$ or $b_{10} \neq 0$, we have only one ring with 21 peaks and in the center the Peregrine breather of order 9.

The study of the solutions to the NLS equation has been done until order $N=$ 6 by Akhmediev et al. in [35] and extrapolated until order $N=10$.

From this present study of order 11, it becomes clear that we can conjecture the structure of solutions to NLS equation.

Precisely, one can partly conjecture the structure of the rogue waves solutions to the NLS equation at the order $N$. Important applications for example in the fields of nonlinear optics and hydrodynamics are made recently; we can cite in particular the works of Akhmediev et al [36] or Kibler et al. [37].

Another interesting study would be to determine which initial conditions can give these types of rogue waves and to discuss the physical excitations which lead to such situations. It would be important to answer this kind of question in the future.

It would be relevant to continue this study to understand these solutions, to try to classify them in the general case of order $N(N>11)$ and to prove the preceding conjectures.

\section{References}

[1] V. E. Zakharov, Stability of periodic waves of finite amplitude on 
a surface of a deep fluid, J. Appl. Tech. Phys, V. 9, 86-94, (1968)

[2] V. E. Zakharov, A.B. Shabat Exact theory of two dimensional self focusing and one dimensinal self modulation of waves in nonlinear media, Sov. Phys. JETP, V. 34, 62-69, (1972)

[3] D. Peregrine, Water waves, nonlinear Schrödinger equations and their solutions, J. Austral. Math. Soc. Ser. B, V. 25, 16-43, (1983)

[4] N. Akhmediev, V. Eleonski, N. Kulagin, Generation of periodic trains of picosecond pulses in an optical fiber : exact solutions, Sov. Phys. J.E.T.P., V. 62, 894-899, (1985)

[5] N. Akhmediev, V. Eleonski, N. Kulagin, Exact first order solutions of the nonlinear Schrödinger equation, Th. Math. Phys., V. 72, N. 2, 183-196, (1987)

[6] N. Akhmediev, A. Ankiewicz, J.M. Soto-Crespo, Rogue waves and rational solutions of nonlinear Schrödinger equation, Physical Review E, V. 80, N. 026601, (2009)

[7] N. Akhmediev, A. Ankiewicz, P.A. Clarkson, Rogue waves, rational solutions, the patterns of their zeros and integral relations, J. Phys. A : Math. Theor., V. 43, 122002-1-9, (2010)

[8] P. Gaillard, Families of quasirational solutions of the NLS equation and multi-rogue waves, J. Phys. A : Meth. Theor., V. 44, $1-15,(2011)$
[9] P. Gaillard, Wronskian representation of solutions of the NLS equation and higher Peregrine breathers, Journal of Math. Sciences : Advances and Applications, V. 13, N. 2, 71-153, (2012)

[10] B. Guo, L. Ling, Q.P. Liu, Nonlinear Schrodinger equation: Generalized Darboux transformation and rogue wave solutions, Phys. Rev. E, V. 85, 026607, (2012)

[11] Y. Ohta, J. Yang, General high-order rogue waves and their dynamics in the nonlinear Schrödinger equation, Pro. R. Soc. A, V. 468, 1716-1740, (2012)

[12] P. Gaillard, Degenerate determinant representation of solution of the NLS equation, higher Peregrine breathers and multi-rogue waves, Jour. Of Math. Phys., V. 54, 013504-1-32, (2013)

[13] P. Gaillard, Multi-parametric deformations of the Peregrine breather of order $\mathrm{N}$ solutions to the NLS equation and multi-rogue waves, Adv. Res., 4, 346-364, 2015

[14] L. Ling, L.C. Zhao, Trajectory Characters of rogue waves, arXiv : 1305.5599v1, 24 May, (2013)

[15] A.A. Gelash, V.E. Zakharov, Superregulier solitonic solutions : a novel scenario for the nonlinear stage of modulation instability, Non linearity, V. 27, 1-39, (2014)

[16] M. Gastineau, J. Laskar, TRIP 1.3.8 : TRIP Reference manual, IMCCE, Paris Observatory, 2014, http://www.imcce.fr/trip/ 
[17] P. Gaillard, Deformations of third order Peregrine breather solutions of the NLS equation with four parameters, Phys. Rev. E, V. 88, 042903-1-9, 2013

[18] P. Gaillard, Six-parameters deformations of fourth order Peregrine breather solutions of the NLS equation, J. Math. Phys., V. 54, 073519-1-22, 2013

[19] P. Gaillard, The fifth order Peregrine breather and its eight-parameters deformations solutions of the NLS equation, Commun. Theor. Phys., V. 61, 365-369, 2014

[20] P. Gaillard, Ten parameters deformations of the sixth order Peregrine breather solutions of the NLS equation, Phys. Scripta, V. 89, 015004-1-7, 2014

[21] P. Gaillard, Higher order Peregrine breathers, their deformations and multi-rogue waves, J. Of Phys. : Conf. Ser., V. 482, 012016-1-7, 2014

[22] P. Gaillard, Other 2N-2 parameters solutions to the NLS equation and $2 \mathrm{~N}+1$ highest amplitude of the modulus of the N-th order AP breather, J. Phys. A: Math. Theor., V. 48, 145203-1-23, 2015

[23] P. Gaillard, M. Gastineau, The Peregrine breather of order nine and its deformations with sixteen parameters solutions of the NLS equation, Phys. Lett. A., V. 379, 13091313, 2015

[24] P. Gaillard, M. Gastineau, Eighteen parameter deformations of the Peregrine breather of order ten solutions of the NLS equation, Int. J. Mod. Phys. C, V. 26, N. 2, 1550016-1-14, 2014

[25] P. Gaillard, V.B. Matveev, Wronskian addition formula and its applications, Max-Planck-Institut für Mathematik, MPI 02-31, V. 161, 2002

[26] P. Gaillard, A new family of deformations of Darboux-Pöschl-Teller potentials, Lett. Math. Phys., V. 68, 77-90, 2004

[27] P. Gaillard, V.B. Matveev, New formulas for the eigenfunctions of the two-particle Calogero-Moser system, Lett. Math. Phys., V. 89, 1-12, 2009

[28] P. Gaillard, V.B. Matveev, Wronskian and Casorai determinant representations for DarbouxPöschl-Teller potentials and their difference extensions, J. Phys A : Math. Theor., V. 42, 1-16, 2009

[29] P. Gaillard, Wronskian representation of solutions of NLS equation and seventh order rogue waves, J. Mod. Phys., V. 4, N. 4, 246-266, 2013

[30] P. Gaillard, V.B. Matveev, Wronskian addition formula and Darboux-Pöschl-Teller potentials, J. Math., V. 2013, ID 645752, 1-10, 2013

[31] P. Gaillard, Two parameters deformations of ninth Peregrine breather solution of the NLS equation and multi rogue waves, J. Math., V. 2013, 1-111, 2013

[32] P. Gaillard, Two-parameters determinant representation of seventh order rogue waves solutions 
of the NLS equation, J. Theor. Appl. Phys., V. 7, N. 45, 1-6, 2013

[33] P. Gaillard, Two parameters wronskian representation of solutions of nonlinear Schrödinger equation, eight Peregrine breather and multi-rogue waves, J. Math. Phys., V. 5, 093506-1-12, 2014

[34] P. Gaillard, Tenth Peregrine breather solution of the NLS, Ann. Phys., V. 355, 293-298, 2015

[35] D. J. Kedziora, A. Ankiewicz, N. Akhmediev, Classifying the hierarchy of the nonlinear Schrödinger equation rogue waves solutions, Phys. Review E, V. 88, 013207-1-12, (2013)

[36] A. Chabchoub, H. Hoffmann, M. Onorato, N. Akhmediev, Super rogue waves : observation of a higher-order breather in water waves, Phys. Review X, V. 2, 16, (2012).

[37] B. Kibler, J. Fatome, C. Finot, G. Millot, F. Dias, G. Genty, N. Akhmediev, J.M. Dudley, The Peregrine soliton in nonlinear fibre optics, Nat, Phys, V. 6, 790795, (2010) 